\title{
CARACTERÍSTICAS QUÍMICAS DE SOLO E RENDIMENTO DE MASSA SECA DE MILHO EM FUNÇÃO DO MÉTODO DE APLICAÇÃO DE FOSFATOS, EM DOIS NÍVEIS DE ACIDEZ ${ }^{(1)}$
}

\author{
P. R. ERNANI ${ }^{(2)}$, C. STECKLING ${ }^{(3)} \&$ C. BAYER ${ }^{(2)}$
}

\begin{abstract}
RESUMO
Apesar da baixa mobilidade do $P$ no solo, os fertilizantes fosfatados estão sendo aplicados sobre a superfície, em alguns sistemas de cultivo. Pouco se conhece sobre a eficiência dessa forma de aplicação, a qual pode ser influenciada pela característica dos fosfatos e pelo pH do solo. E ste trabalho objetivou avaliar o efeito do método de aplicação (superficial ou incorporado) de alguns fertilizantes fosfatados (diamônio fosfato - DAP, superfosfato triplo e fosfato natural ARAD), em dois valores de pH (com e sem calcário), em algumas características químicas do solo e no rendimento de massa seca de milho. Cultivou-se milho durante $\mathbf{2 1}$ dias, em câmara de crescimento, em unidades experimentais com 3,0 kg de solo (base seca). Na ausência de calcário (pH 4,7), a aplicação dos fosfatos na superfície do solo proporcionou maiores rendimentos de massa seca e maior absorção de $\mathbf{P}$ pelo milho do que a incorporação dos fosfatos, sendo o DAP o fertilizante mais eficiente. Na presença de calcário (pH 5,7), não houve diferença entre os métodos de aplicação, e os maiores rendimentos foram obtidos com as fontes solúveis. A aplicação dos fosfatos na superfície do solo aumentou a concentração de $\mathrm{P}$ até $3 \mathrm{~cm}$ de profundidade. $\mathrm{Na}$ ausência de déficit hídrico, o mi lho foi eficiente em absorver o P aplicado sobre a superfície do solo a partir de fontes solúveis em água, mesmo tendo havido baixa mobilidade vertical do nutriente.
\end{abstract}

Termos de indexação: diamônio fosfato, superfosfato triplo, fosfato natural ARAD, acidez do solo.

(1) Realizado com recursos do Programa de Pós-Graduação em Ciência do Solo, Universidade do Estado de Santa Catarina - UDE SC. Recebido para publicação em agosto de 2000 e aprovado em março de 2001.

(2) Professor do Centro de Ciências Agroveterinárias da Universidade do Estado de Santa Catarina - UDESC. Caixa Postal 281, CEP 88520-000 Lages (SC). Bolsista do CNPq. E-mail: prernani@cav.udesc.br

(3) Mestrando em Ciência do Solo, UDESC. 


\title{
SUMMARY: SOIL CHEMICAL CHARACTERISTICS AND CORN DRY MASS YIELD AS AFFECTED BY APPLICATION METHODS OF PHOSPHATE FERTILIZERS, AT TWO SOIL PH LEVELS
}

\begin{abstract}
Phosphorus has very low mobility into the soil, but in spite of this, it has been applied on the soil surface on some soil tillage systems. The efficiency of this form of application is not well known yet but can be affected by the soil pH and type of phosphate applied. This study aimed to assess the effect of the application method (mixed into the soil or on the surface, without incorporation) of phosphate fertilizers (diammonium phosphate, triple superphosphate, and ARAD rock phosphate), at two pH values (with and without lime), on somesoil chemi cal characteristics and on corn dry matter yield. Corn plants weregrown during 21 days after seed emergence in a growth chamber, in experimental units of $3.0 \mathrm{~kg}$ of soil (dry basis). In absence of liming $\left(\mathrm{pH}-\mathrm{H}_{2} \mathrm{O} 4.7\right)$, addition of phosphates on the soil surface gave better yield and higher $\mathrm{P}$ uptake than their incorporation into the soil, and diammonium phosphate was the most efficient source On limed soil $\left(\mathrm{pH}-\mathrm{H}_{2} \mathrm{O} 5.7\right)$, there was no difference between the application methods, and the best yields were obtained with the soluble phosphate sources. Addition of phosphate fertilizers on the soil surface increased $P$ in the soil up to $3 \mathrm{~cm}$ depth. In absence of water deficit, corn plants could obtain enough $\mathrm{P}$ from soluble phosphates added to the soil surface, despite their low mobility.
\end{abstract}

Index terms: diammonium phosphate, triple superphosphate, ARAD rock phosphate, soil acidity, application methods.

\section{NTRODUÇÃO}

Nos solos do sul do Brasil, com acidez el evada e baixa disponi bilidade defósforo, a aplicação de calcário e de fertilizantes fosfatados é fundamental para a obtenção dealtos rendimentos vegetais. A eficiência do $\mathrm{P}$ aplicado ao solo varia de acordo com o tipo de fertilizante fosfatado (Ernani \& Barber, 1990; 1991), com o método de aplicação (M uzilli, 1983; Anghinoni, 1992), ecom a quantidadeaplicada (Anghinoni, 1992; Ernani et. al., 2000), além de características desolo, como pH (Ernani et al., 1996), umidade (Cox \& Barber, 1992), teor de argila (Cox, 1994; Huffman et al., 1996), teor de Ca (Akinremi \& Cho, 1991) eformas deFe (Hernández \& Meurer, 1998).

O método deaplicação dos fertilizantes fosfatados, na superfície ou incorporado ao solo, não tem alterado a absorção de $\mathrm{P}$ eo rendimento das culturas em algumas situações (Muzilli, 1983; Klepker \& Anghinoni, 1996), apesar da baixa mobilidade do $P$ no solo. A boa eficiência do $P$ aplicado sobre a superfície, provavelmente, está relacionada com o maior incremento na concentração de $\mathrm{P}$ na solução do solo nessa região, em conseqüência do aumento na percentagem de saturação dos sítios de adsorção, resultante da redução do contato do fertilizante com o solo. A localização de $\mathrm{P}$, no entanto, diminui o volume de solo fertilizado com o qual as raízes entram em contato, o que pode prejudicar a absorção do nutriente (Zhang \& Barber, 1992), especialmente em períodos de déficit hídrico.

Osfertilizantes fosfatados diferem, principalmente quanto à composição química, solubilidade e cátion acompanhante. Essas diferenças podem influir na taxa de solubilização dos fosfatos e nas reações de precipitação e adsorção química de $\mathrm{P}$ nas regiões adjacentes aos grânulos ou partículas de fosfatos, onde a intensidade é alta, com reflexos na concentração de $\mathrm{P}$ na solução do solo, e com isso influir na eficiência de utilização pelas plantas e no rendimento das culturas (Ernani \& Barber, 1991; Coutinho et al., 1991).

A dissolução do diamônio fosfato(DAP) eleva opH aoredor dos grânul os (Akinremi \& Cho, 1991; Ernani \& Barber, 1991), e com isso diminui a adsorção química de P (Ernani e Barber, 1990; E rnani et al., 1996), a formação de precipitados do $P$ com Fe e Al (Ernani \& Barber, 1991) e a atividade do Al (Ernani $\&$ Barber, 1991).

A dissolução do superfosfato triplo (SFT), por outro lado, diminui o pH ao redor do grânulo, e com isso aumenta a adsorção de $\mathrm{P}$ e a formação de precipitados com Fe e Al (Figueiredo, 1985); como ele é um fosfato de cálcio, também pode originar preci pitados de fósforo com cál cio (Akinremi \& Cho, 1991). A disponibilidade de $P$ a partir da aplicação defosfatos solúveis depende, portanto, da reação que controla o suprimento de $\mathrm{P}$ à solução do solo (adsorção química ou precipitação), do pH ao redor do grânulo e do tipo de precipitado de fósforo que predomina em cada solo.

O comportamento dos fosfatos naturaiséum pouco diferente porque eles dependem primeiramente da solubilização, a qual é favorecida pel o contato com o sol o e pela presença de ${ }^{+}$(He et al., 1996). A partir dela, el es passam a ter o mesmo comportamento que os fosfatos solúveis, porém com menor intensidade, nas reações de preci pitação, em razão das menores 
concentrações de $\mathrm{P}$ nas microrregiões ao redor das partículas de fosfato. $\mathrm{O} \mathrm{pH}$ do solo, especialmente das microrregiões ao redor dos grânulos de fertilizantes, é, portanto, um dos parâmetros de solo com grande influência na disponibilidade de $P$ aos vegetais, por influenciar a solubilidade dos fosfatos naturais (Heet al., 1996) ea magnitude das reações de adsorção e preci pitação do fósforo adicionado ao solo, principalmente na forma de fosfatos solúveis.

Com a crescente utilização do sistema plantio direto, aumenta a importância do conhecimento da magnitude dessas reações, assim como da mobilidade deP no solo, pois os fertilizantes são aplicados sobre a superfície do solo, originando zonas com altas concentrações iônicas.

O presentetrabalho objetivou avaliar a mobilidade do $P$ eas alterações nas características químicas de um Latossolo Alumínico típico resultantes da aplicação de diferentes fertilizantes fosfatados sobre a superfície do solo, bem como o seu efeito no rendimento de massa seca e na absorção de $\mathrm{P}$ por plântulas de milho, em dois valores de $\mathrm{pH}$.

\section{MATERIAL E MÉTODOS}

A presente pesquisa consistiu em dois experimentos realizados no ano de 1998, no Centro Agroveterinário da UDESC, em Lages (SC).

Um dos experimentos foi feito em câmara de crescimento, utilizando-se o milho como planta-teste e amostras de solo com estrutura deformada, coletadas no horizonte $\mathrm{A}$ de um Latossolo Bruno Alumínico Típico, que apresentava $\mathrm{pH}-\mathrm{H}_{2} \mathrm{O}=4,7$, $\mathrm{pH}-\mathrm{CaCl}_{2}=4,3, \mathrm{Al}^{3+}=17 \mathrm{mmol}_{\mathrm{c}} \mathrm{kg}^{-1}, \mathrm{P}=2 \mathrm{mg} \mathrm{kg}^{-1}$ Mehlich-1, argila $=550 \mathrm{~g} \mathrm{~kg}^{-1}$ e MO $=40 \mathrm{~g} \mathrm{~kg}^{-1}$.

Os tratamentos consistiram de um fatorial com três fertilizantes fosfatados (diamônio fosfato, superfosfato tripl o e o fosfato natural ARAD), além de uma testemunha sem $P$, dois métodos de aplicação (sobre a superfície ou incorporado ao solo), e dois níveis de acidez (com esem cal cário). Três repetições foram distribuídas no delineamento experimental completamente casualizado.

Nos tratamentos com calagem, aplicou-se o equivalente a $10 \mathrm{t} \mathrm{ha}^{-1}$ de calcário dolomítico comercial, corrigido para PRNT $100 \%$. A seguir, todas as unidades experimentais, com $3,0 \mathrm{~kg}$ de solo seco cada, foram incubadas por 25 dias, em temperatura ambiente, com umidade de aproximadamente $80 \%$ da capacidade de retenção de água. Após esse período, as amostras de solo apresentaram $\mathrm{pH}$ $\mathrm{H}_{2} \mathrm{O}$ 5,7 e foram transferidas para tubos de PVC, com $10 \mathrm{~cm}$ de diâmetro e $38 \mathrm{~cm}$ de altura, quando se aplicaram os tratamentos de P. O P foi aplicado na dose total de $80 \mathrm{mg} \mathrm{kg}^{-1}$ (187 $\mathrm{mg} \mathrm{kg}^{-1}$ de $\mathrm{P}_{2} \mathrm{O}_{5}$ ); nos tratamentos com superfosfato triplo (SFT), fosfato natural e, na testemunha, aplicou-se a mesma quantidade de $\mathrm{N}$ adicionado pelo DAP $\left(90 \mathrm{mg} \mathrm{kg}^{-1}\right.$ deN), na forma deuréia líquida. Cultivaram-se duas plantas de milho (Cargill 855) por unidade experimental, durante 21 dias após a emergência, em câmara de crescimento, ajustada para a temperatura de $28 \% \mathrm{C}$ e umidade relativa do ar de $85 \%$.

Para facilitar a separação do solo em camadas, nas unidades experimentais onde o $P$ foi aplicado na superfície do solo, cada tubo (com $100 \mathrm{~mm}$ de diâmetro) foi construído, de cima para baixo, pela justaposição de três anéis com $1 \mathrm{~cm}$ de altura, dois com 2,5 cm, um com $10 \mathrm{~cm}$, e o último com $20 \mathrm{~cm}$. Nos tratamentos em que o P foi incorporado ao solo, cada tubo foi formado por um anel superior com $20 \mathrm{~cm}$ de altura (porção do solo em que os fertilizantes foram incorporados), seguido por três com $1 \mathrm{~cm}$, dois com 2,5 cm, e o último com $10 \mathrm{~cm}$. A parte inferior dos tubos foi vedada por uma tampa dePVC, ea superfície do sol o foi coberta com plástico preto, para amenizar a perda de água por evaporação. Água destilada foi adicionada a cada dois dias, num volume de $50 \mathrm{ml}$ por tubo.

Nas plantas de milho, determinaram-se a massa seca da parte aérea e a concentração de P no tecido, efoi calculada a quantidade de P absorvido. O tecido vegetal foi digerido com solução nitroperclórica (Tedesco et al., 1995) e a determinação do P feita em espectrofotômetro de emissão induzida por plasma (ICP). Nas amostras de solo, coletadas no final do experimento, em todas as profundidades del imitadas pelos anéis, determinaram-se $\mathrm{pH}, \mathrm{Ca}, \mathrm{Mg}$ e $\mathrm{Al}$ trocáveiseP extraível. O pH foi determinado em água e em solução de $\mathrm{CaCl}_{2} \quad 0,01 \mathrm{~mol} \mathrm{~L}^{-1}$, numa relação solo/solvente de 1:1; Ca, Mg eAl foram extraídos com solução de $\mathrm{KCl} 1,0 \mathrm{~mol} \mathrm{~L}^{-1}$ e determinados por ICP; e o P foi extraído com solução de Mehlich-1 e com resina trocadora deânions, sendo ambos determinados por colorimetria (Tedesco et al., 1995). Em todas as extrações, usou-se uma relação sol o/sol vente de 1:10.

O segundo experimento foi realizado com o objetivo de elucidar alguns resultados obtidos no experimento com plantas. N ele, tentou-se simular o efeito ocasionado por alguns fertilizantes no $\mathrm{pH}$ do solo das regiões adjacentes aos grânulos. Como as variações ocorrem apenas em microrregiões, e por isso são difíceis de ser quantificadas, optou-se por misturar o sol o com uma quantidade extremamente grande de fertilizante com vistas em obter valores mensuráveis num volume expressivo de solo. Para isso, misturaram-se $200 \mathrm{~g}$ de uréia, SFT ou DAP com amostras de $1,0 \mathrm{~kg}$ de solo (base seca). A seguir, as unidades experimentais, com três repetições, foram incubadas por 32 dias, em temperatura ambiente e com umidadedeaproximadamente $80 \%$ da capacidade de retenção de água. Amostras para determinação do $\mathrm{pH}$ foram coletadas 1, 2, 4, 8, 16 e 32 dias após a aplicação dos tratamentos. $\mathrm{O}$ pH foi determinado, usando-se água como solvente, na relação 1:1. O solo utilizado foi o mesmo do experimento anterior, porém coletado aproximadamente seis meses após. 
A análise estatística dos efeitos dos tratamentos sobre as variáveis consistiu, no experimento I, na análise da variância. A diferença entreas médias de tratamentos foi avaliada pel o teste de Duncan, a 5\%. Os resultados referentes ao experimento II não foram analisados estatistiscamente, observando-se apenas as tendências dos efeitos dos fertilizantes no $\mathrm{pH}-\mathrm{H}_{2} \mathrm{O}$ do solo.

\section{RESULTADOS E DISCUSSÃO}

$\mathrm{O}$ pH do solo não foi afetado pelos fertilizantes fosfatados nem pela sua forma de aplicação, em nenhuma profundidade (dados não apresentados). O Al trocável, entretanto, diminuiu nas camadas superficiais do solo, até, no máximo, três centímetros de profundidade, dependendo do fertilizanteaplicado (Quadro 1). No sol o onde foram aplicados SFT, ARAD, e na testemunha (sem $P$ ), os valores de Al foram menores nos $3 \mathrm{~cm}$ superficiais e não houve diferença entre os tratamentos. Onde se aplicou DAP, o Al diminuiu somente no centímetro superficial e, mesmo assim, foi maior que na testemunha. A diminuição do Al trocável no solo que recebeu os fertilizantes fosfatados poderia ter sido causada pela preci pitação do Al com os fosfatos (Figueiredo, 1985; Akinremi \& Cho, 1991), porque os fertilizantes não alteraram o pH médio do solo.

Entretanto, como o Al diminuiu também na testemunha, onde não se aplicou $P$, outro fenômeno aconteceu, possivelmente relacionado com a liberação de exsudatos a partir das raízes concentradas nessas camadas superficiais. Quando os fertilizantes foram incorporados ao solo, o Al trocável não foi afetado na camada fertilizada $(20 \mathrm{~cm})$, possivelmente pela diluição do efeito das reações pela mistura com o solo (dados não apresentados).
Os fertilizantes fosfatados alteraram os valores de Ca nas camadas superficiais, mas não os de $\mathrm{Mg}$ (Quadro 2). A aplicação deSFT eARAD na superfície do solo aumentou o Ca trocável somente no centímetro superficial, porém apenas na ausência de calagem; o DAP não influiu nos teores de $\mathrm{Ca}$ (Quadro 2). Para solos deficientes em Ca, portanto, os fosfatos de cálcio teriam a vantagem de suprir Ca. Para sol os cal cariados, ou com bom suprimento natural de Ca, no entanto, a eficiência dos fosfatos dependerá do tipo de precipitado de fósforo predominante, pois um aumento dos valores de $\mathrm{Ca}$ poderá resultar em maior precipitação desse nutriente com o fósforo (Akinremi \& Cho, 1991).

A mobilidade de $P$ no solo foi baixa, o que proporcionou grande acúmulo na superfície do solo. A aplicação dos fosfatos na superfície acumulou P até à profundidade de $3 \mathrm{~cm}$, independentemente da calagem, e a maior mobilidade ocorreu no sol o que recebeu SFT (Quadro 3). Na camada de 0-1,0 cm, o P variou de $3 \mathrm{mg} \mathrm{kg}^{-1}$, na testemunha, até $430 \mathrm{mg} \mathrm{kg}^{-1}$, no tratamento com SFT. Nessa camada, o P chegou a ser 140 vezes maior do que nas camadas de solo quenão foram influenciadas pela adição dos fosfatos. Apesar da baixa mobilidade, o $\mathrm{P}$ aplicado sobre a superfície desceu al guns centímetros, possivel mente pela sua grande concentração em superfície, com provável saturação dos sítios de adsorção. Em condições de campo, onde o sol o não é mobilizado, a descida de $\mathrm{P}$ deverá ser menor, considerando a maior densidade do sol o no campo relativamente à do solo existente nos vasos.

A concentração de $P$ variou com o tipo de fosfato aplicado. É provável que os maiores valores de P, onde se aplicou SFT, e nas unidades experimentais sem calcário relativamente às cal cariadas, sejam devidos à maior absorção de $\mathrm{P}$ pelas plantas nos tratamentos com DAP e naqueles calcariados (Quadro 5), uma vez que as amostras de sol o foram coletadas após o período de 21 dias de cultivo do milho.

Quadro 1. Teores de Al trocável, no tratamento sem calcário, considerando a aplicação dos fertilizantes fosfatados na superfície do solo

\begin{tabular}{|c|c|c|c|c|c|c|c|c|}
\hline \multirow{2}{*}{ Fontes de P } & \multicolumn{8}{|c|}{ Profundidade } \\
\hline & $0,0-1,0$ & $1,0-2,0$ & $2,0-3,0$ & $3,0-5,5$ & $5,5-8,0$ & $8,0-18,0$ & $18,0-38,0$ & Média \\
\hline & \multicolumn{8}{|c|}{$\longrightarrow \mathrm{mmol}_{\mathrm{c}} \mathrm{kg}^{-1}$} \\
\hline Sem $P$ & $6 a \mathrm{~B}$ & $9 \mathrm{bB}$ & $11 \mathrm{bB}$ & $16 \mathrm{CA}$ & $17 \mathrm{c}$ & $17 \mathrm{c}$ & $16 c$ & 16 \\
\hline SFT & $3 a \mathrm{~B}$ & $10 \mathrm{~b} \mathrm{~B}$ & 12 bcB & $14 \mathrm{cdB}$ & $17 \mathrm{~d}$ & $17 d$ & $16 d$ & 16 \\
\hline DAP & 10 a A & $19 \mathrm{cdA}$ & $19 \mathrm{~cd} A$ & 17 bcdA & $18 \mathrm{bcd}$ & $16 \mathrm{bc}$ & $15 \mathrm{~b}$ & 15 \\
\hline ARAD & $3 a B$ & $7 \mathrm{~b} \mathrm{~B}$ & $10 \mathrm{bcB}$ & $12 \mathrm{cdB}$ & $15 \mathrm{~d}$ & $15 d$ & $14 \mathrm{~d}$ & 14 \\
\hline
\end{tabular}

Médias seguidas pela mesma letra maiúscula, nas colunas, indicam ausência de diferença entre as fontes de $\mathrm{P}$ dentro da mesma profundidade; médias seguidas pela mesma letra minúscula, nas linhas, indicam ausência de diferença entre as profundidades dentro do mesmo tratamento de P; ausência de letras indica inexistência de diferença pelo teste de Duncan a 5\%. 
Quadro 2. Teores de Ca e Mg trocáveis em três profundidades do solo $(0-1,1,0-2,0$ e 2,0-38,0 cm), considerando a aplicação dos fertilizantes fosfatados na superfície do solo e a pH do solo

\begin{tabular}{|c|c|c|c|c|c|c|}
\hline \multirow{2}{*}{ Fontes de $P$} & \multicolumn{3}{|c|}{$\mathrm{Ca}$} & \multicolumn{3}{|c|}{ Mg } \\
\hline & $0,0-1,0$ & $1,0-2,0$ & $2,0-38,0$ & $0,0-1,0$ & $1,0-2,0$ & $2,0-38,0$ \\
\hline & \multicolumn{6}{|c|}{$-\mathrm{mmol}_{\mathrm{c}} \mathrm{kg}^{-1}$} \\
\hline & \multicolumn{6}{|c|}{ Sem calcário $\left(\mathrm{pH}-\mathrm{H}_{2} \mathrm{O} 4,7\right)$} \\
\hline $\begin{array}{l}\text { Sem P } \\
\text { SFT } \\
\text { DAP } \\
\text { ARAD }\end{array}$ & $\begin{array}{l}12 \mathrm{~B} \\
40 \mathrm{aA} \\
13 \mathrm{~B} \\
39 \mathrm{aA}\end{array}$ & $\begin{array}{l}12 \\
18 b \\
10 \\
19 b\end{array}$ & $\begin{array}{l}12 \\
12 b \\
11 \\
11 b\end{array}$ & $\begin{array}{l}12 \\
15 \\
16 \\
11\end{array}$ & $\begin{array}{l}12 \\
12 \\
11 \\
11\end{array}$ & $\begin{array}{l}12 \\
12 \\
12 \\
11\end{array}$ \\
\hline ARAD & \multicolumn{6}{|c|}{ Com cal cário $\left(\mathrm{pH}-\mathrm{H}_{2} \mathrm{O} 5,7\right)$} \\
\hline $\begin{array}{l}\text { Sem P } \\
\text { SFT } \\
\text { DAP } \\
\text { ARAD }\end{array}$ & $\begin{array}{l}39 \mathrm{BC} \\
45 \mathrm{aB} \\
43 \mathrm{~B} \\
53 \mathrm{aA}\end{array}$ & $\begin{array}{l}41 \\
38 b \\
41 \\
45 b\end{array}$ & $\begin{array}{l}43 \\
39 b \\
41 \\
36 b\end{array}$ & $\begin{array}{l}38 \\
40 \\
44 \\
39\end{array}$ & $\begin{array}{l}40 \\
42 \\
38 \\
40\end{array}$ & $\begin{array}{l}43 \\
41 \\
42 \\
44\end{array}$ \\
\hline
\end{tabular}

Médias seguidas pela mesma letra maiúscula, nas colunas, indicam ausência de diferença entre as fontes de $\mathrm{P}$ dentro da mesma profundidade; médias seguidas pela mesma letra minúscula, nas linhas, indicam ausência de diferença entre as profundidades dentro do mesmo tratamento de P; ausência de letras indica inexistência de diferença pelo teste de Duncan a 5\%.

Quadro 3. Teores de P extraível pelo método da resina, nas diferentes profundidades do solo, considerando a aplicação de fertilizantes fosfatados na superfície do solo

\begin{tabular}{|c|c|c|c|c|c|c|c|c|}
\hline \multirow{2}{*}{ Profundidade } & \multicolumn{4}{|c|}{ Sem calcário (pH-H $\left.\mathrm{H}_{2} \mathrm{O}, 7\right)$} & \multicolumn{4}{|c|}{ Com calcário (pH-H 20 5,7) } \\
\hline & DAP & SFT & ARAD & Sem $P$ & DAP & SFT & ARAD & Sem $P$ \\
\hline $\mathrm{cm}$ & \multicolumn{8}{|c|}{$-\mathrm{mg} \mathrm{kg}^{-1}$} \\
\hline $0,0-1,0$ & $209 a$ & $430 a$ & $66 a$ & 3 & $185 a$ & $355 a$ & $29 a$ & 4 \\
\hline $1,0-2,0$ & $28 \mathrm{~b}$ & $46 \mathrm{~b}$ & $27 \mathrm{~b}$ & 4 & $23 b$ & $43 \mathrm{~b}$ & $18 a b$ & 5 \\
\hline $2,0-3,0$ & $10 \mathrm{c}$ & $17 \mathrm{c}$ & $6 c$ & 3 & $10 \mathrm{bc}$ & $19 \mathrm{c}$ & 8 bc & 5 \\
\hline $3,0-5,5$ & $6 c$ & $8 \mathrm{~cd}$ & $4 c$ & 3 & $6 c$ & $8 \mathrm{~cd}$ & $3 c$ & 5 \\
\hline $5,5-8,0$ & $4 \mathrm{C}$ & $5 \mathrm{~cd}$ & $4 c$ & 2 & $4 c$ & $5 d$ & $3 c$ & 3 \\
\hline $8,0-18,0$ & $3 c$ & $3 d$ & $3 c$ & 2 & $3 c$ & $3 d$ & $3 c$ & 4 \\
\hline $18,0-38,0$ & $2 c$ & $2 d$ & $2 c$ & 2 & $2 c$ & $4 d$ & $4 \mathrm{bc}$ & 4 \\
\hline Média & $20 \mathrm{~B}$ & $39 \mathrm{~A}$ & $10 \mathrm{C}$ & $3 \mathrm{D}$ & $19 \mathrm{~B}$ & $34 \mathrm{~A}$ & $7 \mathrm{C}$ & $4 C$ \\
\hline
\end{tabular}

Médias seguidas pela mesma letra maiúscula, nas linhas, indicam ausência de diferença entre as fontes de $\mathrm{P}$ dentro da mesma profundidade; médias seguidas pela mesma letra minúscula, nas colunas, indicam ausência de diferença entre as profundidades dentro do mesmo tratamento de P; ausência de letras indica inexistência de diferença pelo teste de Duncan a 5\%.

Os menores val ores de $\mathrm{P}$ no solo ocorreram onde se aplicou ARAD, decorrente da menor solubilização desse fosfato natural relativamente aos fosfatos solúveis. Quando os fosfatos foram incorporados ao solo, o P extraível não diferiu entre as fontes de $\mathrm{P}$ (Quadro 4), ou entre as profundidades, graças à diluição do $\mathrm{P}$ aplicado na camada de $20 \mathrm{~cm}$ e à alta capacidade de retenção de P por esse solo (Ernani et al., 1996).

$\mathrm{O}$ acúmulo de $\mathrm{P}$ nos primeiros centímetros superficiais, decorrente da aplicação dos fosfatos sobre a superfície, alerta para a importância da existência de umidade e de raízes nesses locais, as quais são estimuladas pela presença de P (Lu \& Miller, 1994; Zhang \& Barber, 1992). Além disso, mostra que, em virtude da alta concentração de $P$ nessas regiões, as reações de precipitação devem assumir maior importância que as de adsorção química. Mostra, ainda, a importância da coleta de amostras de solo em pequenas profundidades as quais revelam diferenças que nao aparecem quando camadas mais espessas de sol o são amostradas. 
Quadro 4. Teores de P extraível pelos métodos de Mehlich-1 e da resi na trocadora de ânions, considerando o método de aplicação dos fertilizantes fosfatados

\begin{tabular}{|c|c|c|c|c|}
\hline \multirow{2}{*}{ Fonte de $P$} & \multicolumn{2}{|c|}{ Resina } & \multicolumn{2}{|c|}{ Mehlich-1 } \\
\hline & Superficial & Incorporado & Superficial & Incorporado \\
\hline & \multicolumn{4}{|c|}{$\longrightarrow \mathrm{mg} \mathrm{kg}^{-1}$} \\
\hline Sem $P$ & $3,1 \mathrm{~d}$ & 3,1 & $2,3 d$ & 2,8 \\
\hline SFT & $15,4 \mathrm{a}$ & 3,3 & $13,2 \mathrm{~b}$ & 3,4 \\
\hline DAP & $8,8 \mathrm{~b}$ & 3,0 & $7,9 c$ & 4,1 \\
\hline ARAD & $5,0 \mathrm{cB}$ & 3,0 & $22,0 \mathrm{aA}$ & 4,8 \\
\hline
\end{tabular}

Os métodos deextração deP do sol o quantificaram valores diferentes, principalmente para o fosfato natural (Quadro 4). O solo que recebeu o fosfato natural de ARAD sobre a superfície apresentou aproximadamente 4,5 vezes mais $P$ pelo método de Mehlich em relação ao método da resina, isso porque o primeiro utiliza uma solução extratora ácida, que dissolve as partículas de fosfato de cálcio do fertilizante queainda não haviam dissolvido no solo, superestimando o $\mathrm{P}$ para as plantas. $\mathrm{O}$ método da resina trocadora de ânions, diferentemente, quantifica apenas as frações mais lábeis do P do solo, que já dissolveram. Para os fosfatos solúveis, o método de extração não influenciou os valores de $P$ no solo (Quadro 4).

A calagem aumentou o rendimento demassa seca do milho e a absorção de $\mathrm{P}$, bem como influenciou a eficiência dos métodos de aplicação dos fosfatos (Quadro 5). Nos tratamentos que receberam cal cário, o rendimento e a absorção de $\mathrm{P}$ não foram influencia-dos pelos métodos de aplicação dos fertilizantes fosfatados, possivel mente, pel o aumento na disponibilidade do $P$ nativo causado pela calagem (Ernani et al., 1996; Ernani et al., 2000), pela menor adsorção química do $\mathrm{P}$ incorporado (Anghinoni, 1992) e pela eliminação da fitotoxidez do Al (Ernani \& Barber, 1990; 1991; Santos et al., 1996).

$\mathrm{Na}$ ausência de calcário, entretanto, a aplicação dos fosfatos na superfície do solo foi mais eficiente em aumentar o rendimento de massa seca e a absorção deP do quea incorporação, provavel mente pela menor retenção deP pela fase sól ida (Anghinoni, 1992), dado o menor contato dos fertilizantes com o solo. A aplicação localizada dos fosfatos satura os sítios de adsor ção de P nos centímetros superficiais, e, com isso, proporciona alta concentração de $P$ na solução do sol o nessas zonas, favorecendo a absorção de $P$ pelas raízes que se encontram nesses locais (Castilhos \& Anghinoni, 1988; Lu \& Miller, 1994).
Os efeitos benéficos da localização de $\mathrm{P}$ no solo, entretanto, parecem diminuir com o tempo, mesmo dentro do ciclo de desenvolvimento de uma cultura (Anghinoni, 1992), razão por que a eficiência da local ização dependerá de quanto $\mathrm{P}$ a cultura absorve nos períodos iniciais de desenvolvimento. Em experimentos de campo, Muzilli (1983), eKlepker \& Anghinoni (1996) observaram que o rendimento de culturas anuais não sofreu al teração com a apl icação dos fertilizantes fosfatados.

A eficiência dos fosfatos em aumentar o rendimento de matéria seca variou com o pH do solo (calagem) e com o método de aplicação (Quadro 5). $\mathrm{Na}$ presença de calcário ( $\mathrm{pH} 5,7)$, o DAP foi o fertilizante fosfatado mais eficiente em aumentar o rendimento demassa seca do milho, nos dois métodos de aplicação deP, seguido peloSFT, eeste peloARAD, que não diferiu da testemunha. Na ausência de calcário ( $\mathrm{pH} 4,7)$, quando os fertilizantes foram aplicados na superfície do solo, os maiores rendimentos foram obtidos com o DAP e com o SFT, seguido pelo ARAD que não diferiu do SFT, e, por último, pela testemunha, sem $P$; quando el es foram incorporados ao solo, o rendimento foi muito baixo, possivel mente limitado pela alta fitotoxidez de Al e pela grande capacidade de adsorção de $\mathrm{P}$ por esse solo, e nenhum fertilizante aumentou o rendimento de massa seca domilho. A boa performancedoARAD no sol o sem cal cário relativamente ao sol o cal cariado deve-se provavel menteà maior dissol ução do fosfato natural em condições de baixo $\mathrm{pH}$.

A maior eficiência do DAP em aumentar o rendimento de matéria seca de milho relativamente aos demais fosfatos provavel mente está relacionada com a elevação do pH ao redor de seus grânulos, fenômeno esseanteriormente observado por Ernani \& Barber (1991). No experimento sem plantas, onde se tentou simular o efeito dos fertilizantes sobre o $\mathrm{pH}$ do solo das regiões adjacentes aos grânulos, 
Quadro 5. Rendimento de massa seca do milho e P absorvido, considerando o pH do solo e o método de aplicação de fertilizantes fosfatados

\begin{tabular}{|c|c|c|c|c|c|}
\hline \multirow{2}{*}{ F osfato } & \multicolumn{2}{|c|}{ Sem calcário (pH 4,7) } & \multicolumn{2}{|c|}{ Com calcário (pH 5,7) } & \multirow{2}{*}{ Média } \\
\hline & P superfície & P incorporado & P superfície & P incorporado & \\
\hline & & 1 & a seca ( $g$ vaso- & & 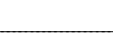 \\
\hline $\begin{array}{l}\text { Sem P } \\
\text { DAP } \\
\text { SFT } \\
\text { ARAD }\end{array}$ & $\begin{array}{l}0,48 \mathrm{c} \\
1,22 \mathrm{a} \\
0,95 \mathrm{ab} \\
0,85 \mathrm{~b}\end{array}$ & $\begin{array}{l}0,48 \\
0,46 \\
0,70 \\
0,57\end{array}$ & $\begin{array}{l}0,65 \mathrm{c} \\
1,72 \mathrm{a} \\
1,26 \mathrm{~b} \\
0,74 \mathrm{c}\end{array}$ & $\begin{array}{l}0,65 \mathrm{c} \\
1,76 \mathrm{a} \\
1,23 \mathrm{~b} \\
0,74 \mathrm{c}\end{array}$ & $\begin{array}{l}0,53 \mathrm{c} \\
1,29 \mathrm{a} \\
1,04 \mathrm{~b} \\
0,73 \mathrm{c}\end{array}$ \\
\hline Média métodos & $0,87 \mathrm{~A}$ & $0,55 \mathrm{~B}$ & 1,09 & 1,09 & \\
\hline Média calagem & \multicolumn{2}{|c|}{$0,71 \mathrm{~B}$} & \multicolumn{2}{|c|}{$1,09 \mathrm{~A}$} & \\
\hline $\begin{array}{l}\text { Sem P } \\
\text { DAP } \\
\text { SFT } \\
\text { ARAD }\end{array}$ & $\begin{array}{l}0,65 \mathrm{~b} \\
2,36 \mathrm{a} \\
1,32 \mathrm{~b} \\
1,20 \mathrm{~b}\end{array}$ & $\begin{array}{l}0,65 \\
1,15 \\
1,12 \\
0,82\end{array}$ & $\begin{array}{l}1,02 \mathrm{~b} \\
2,39 \mathrm{a} \\
1,84 \mathrm{a} \\
1,14 \mathrm{~b}\end{array}$ & $\begin{array}{l}0,90 \mathrm{c} \\
2,73 \mathrm{a} \\
1,76 \mathrm{~b} \\
1,13 \mathrm{bc}\end{array}$ & $\begin{array}{l}0,89 \mathrm{c} \\
2,15 \mathrm{a} \\
1,52 \mathrm{~b} \\
0,99 \mathrm{c}\end{array}$ \\
\hline Média métodos & $1,38 \mathrm{~A}$ & $0,93 \mathrm{~B}$ & 1,60 & 1,63 & \\
\hline Média calagem & \multicolumn{2}{|c|}{$1,16 \mathrm{~B}$} & \multicolumn{2}{|c|}{$1,62 \mathrm{~A}$} & \\
\hline
\end{tabular}

Médias seguidas pela mesma letra, maiúsculas, nas linhas, indica diferença entre métodos de aplicação ou de calagem, e minúsculas, nas colunas, indicam diferença entre fontes de $\mathrm{P}$ dentro de cada método de aplicação; ausência de letras indica inexistência de diferença pelo teste de Duncan a 5\%.

verificou-se que a aplicação de uma alta dose de fertilizantes alterou o pH do solo. A uréia e o DAP el evaram opH de 5,2 para 7,0 e 6,8, respectivamente, e o SFT baixou o pH para 4,0, na média das seis determinações, realizadas nos 32 primeiros dias após a mistura dos fertilizantes com o solo (Figura 1). Com a uréia e o DAP, o pH oscilou com o tempo: diminuiu do primeiro para o segundo dia e, a partir daí, aumentou atéo décimo sexto dia, quando vol tou a diminuir, porém sempre mantendo-se mais alto que no sol o que não recebeu nenhum fertilizante.

$\mathrm{Na}$ testemunha e no sol o tratado com SFT, o pH foi aproximadamente constante aolongo dos 32 dias avaliados. A hidrólise da uréia produz hidroxilas e bicarbonatos, que reagem com o $\mathrm{H}^{+}$nas regiões adjacentes aos grânulos, el evando inicial menteo pH do solo em contato com a respectiva solução. Posteriormente, com a nitrificação, o pH baixa, e isso ocorreu a partir do décimo-sexto dia após a mistura dos fertilizantes com o solo. A dissociação do DAP também consome $\mathrm{H}^{+}$inicialmente (Ernani \& Barber, 1990; 1991), pois algumas moléculas de $\mathrm{HPO}_{4}{ }^{2-}$ transformam-se em $\mathrm{H}_{2} \mathrm{PO}_{4}^{-}$nos valores de $\mathrm{pH}$ comumenteencontrados em solos. Com oSFT, ocorre o contrário, pois sua dissol ução li bera íons $\mathrm{H}^{+}$a partir do $\mathrm{H}_{2} \mathrm{PO}_{4}^{-}$(Figueiredo, 1985). Em condições de campo, essas al terações de pH ocorrem somente nas regiões adjacentes aos grânulos, mas podem ser importantes, principalmente nos períodos iniciais subseqüentes à aplicação dos fertilizantes, onde a magnitude das reações é grande.

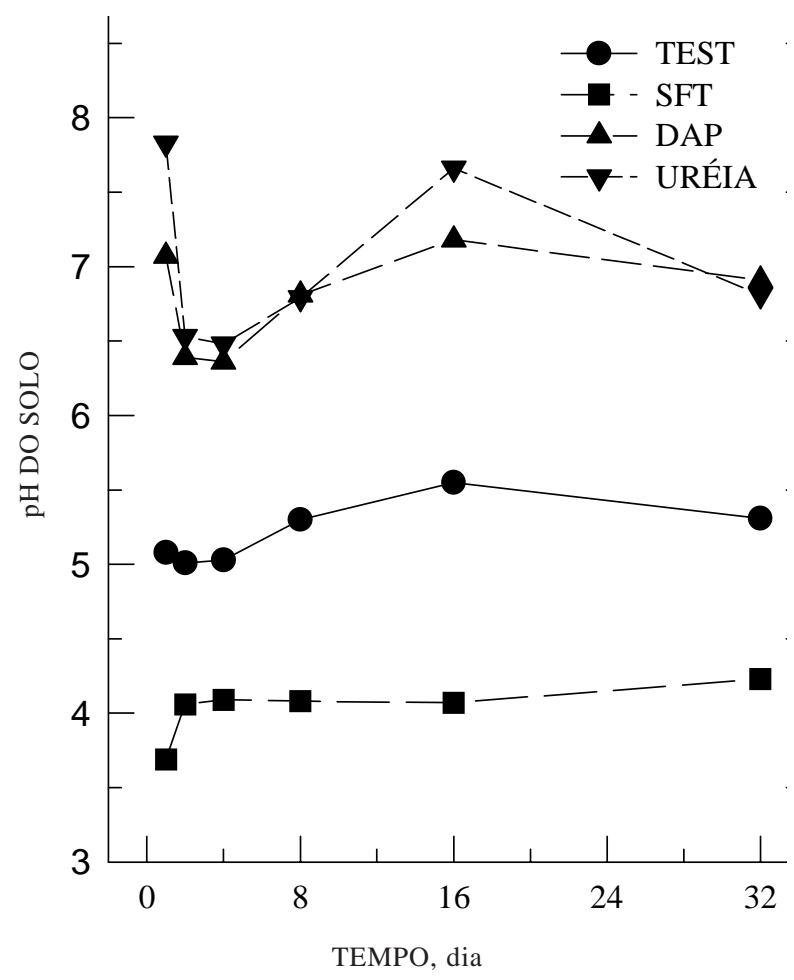

Figura 1. Valores de pH do solo em diferentes épocas após a mistura de uma alta dose $(20 \%$ em peso) de superfosfato triplo (SFT), diamônio fosfato (DAP), ou uréia com um Latossolo Bruno. Média de três repetições. 


\section{CONCLUSÕES}

1. O pH do solo influenciou a eficiência dos métodos de aplicação e das fontes de fósforo.

2. E m condições de alta capacidade de retenção de P, a aplicação localizada dos fosfatos foi mais eficiente que a incorporação dos fosfatos ao solo.

3. A aplicação dos fertilizantes fosfatados sobre a superfície concentrou oP, diminuiu AI trocável até, no máximo, três centímetros de profundidade.

4. No estádio inicial de desenvolvimento, sem limitação hídrica, o mil ho demonstrou habilidadeem absorver o P aplicado sobre a superfície do solo, apesar da baixa mobilidade vertical dessenutriente.

\section{LITE RATURA CITADA}

AKINREMI, O.O. \& CHO, C.M. Phosphate transport in calciumsaturated systems: II. Experimental results in a model system. Soil Sci. Soc. Am. J., 55:1282-1287, 1991.

ANGHINONI, I. Uso de fósforo pelo milho afetado pela fração de solo fertilizada com fosfato solúvel. R. Bras. Ci. Solo, 16:349-353, 1992.

CASTILHOS, D.D \& ANGHINONI, I I Influência do suprimento de fósforo a diferentes frações do sistema radicular sobre o comportamento do milho. R. Bras. Ci. Solo, 12:263-267, 1988.

COUTINHO, E.L.M.; NATALE, W.;VILLA NOVA, A.S. \& SITTA, D.S.X. E ficiência agronômica defertilizantes fosfatados para a cultura da soja. Pesq. Agropec. Bras., 26:1393-1399, 1991.

COX, F.R. Predicting increases in extractable phosphorus from fertilizing soils of varying clay content. Soil Sci. Soc. Am. J ., 58:1249-1253, 1994

COX, M.S. \& BARBER, S.A. Soil phosphorus level for equal P uptake from four soils with different water contents at the same water potential. Plant Soil, 143:93-98, 1992.

ERNANI, P.R. \& BARBER, S.A. Comparison of P-availability from monocalcium and diammonium phosphates using a mechanistic nutrient uptake model. Fert. Res., 22:15-20, 1990.
ERNANI, P.R. \& BARBER, S.A. Corn growth and changes of soil and root parameters as affected by phosphate fertilizers and liming. Pesq. Agropec. Bras., 26:1309-1314, 1991.

ERNANI, P.R.; FIGUEIREDO, O.R.A.; BECEGATO, V. \& ALMEIDA, J .A. Decréscimo da retenção de fósforo no solo pelo aumento do pH. R. Bras. Ci. Solo, 20:159-162, 1996.

ERNANI, P.R.; NASCIMENTO, J.A.L.; CAMPOS, M.L. \& CAMILLO, R.J. Influencia da combinação de fósforo e calcário no rendimento de. R. Bras. Ci. Solo, 24:537-544, 2000.

FIGUEIREDO, O.A.R. Reações de superfosfato triplo e de cama de galinha poedeira com um solo Latossolo Bruno. Porto Algre, Universidade Federal do Rio Grande do Sul, 1985. 85p. (Tese de Mestrado)

HE, Z.L.; BALIGAR, V.C.; MARTENS, D.C.; RITCHEY, K.D. \& KEMPER,W.D. Factors affecting phosphate rock dissolution in acid soil amended with liming materials and cellulose. Soil Sci. Soc. Am. J ., 60:1596-1601, 1996.

HERNANDEZ, J . \& MEURER, E. Adsorção de fósforo e sua relação com formas de ferro em dez solos do U ruguai. R. Bras. Ci. Solo, 22:223-230, 1998.

HUFFMAN, S.A.; COLE, C.V. \& SCOTT, N.A. Soil texture and residue addition affects on soil phosphorus transformation. Soil Sci. Soc. Am. J ., 60:1095-1101, 1996.

KLEPKER, D. \& ANGHINONI, I. Modos de adubação, absorção de nutrientes e rendimento de milho em diferentes preparos do solo. Pesq. Agropec. Gaúcha, 2:79-86, 1996.

LU, S. \& MILLER, M.H. Prediction of phosphorus uptake by fieldgrown maize with the Barber-Cushman model. Soil Sci. Soc. Am. J., 58:852-857, 1994

MUZI LLI , O. I nfluência do sistema de plantio direto, comparado ao convencional, sobre a fertilidade da camada arável do solo. R. Bras. Ci. Solo, 7:95-102, 1983.

SANTOS, J.C.P.; KAMINSKI, J .; ERNANI, P.R. \& MAFRA, A.L. Rendimento de massa seca e absorção de fósforo pel o milho afetado pela aplicação de fósforo, cal cário e incubação com fungos micorrízicos. Ci. Rural, 26:63-67, 1996.

TEDESCO, M.J .; GIANELLO, C.; BISSANI, C.A.; WOLKWEISS, S.J. \& BOHNEN, H. Análise do solo, plantas e outros materiais. 2.ed. Porto Alegre, Universidade Federal do Rio Grande do Sul, 1995. (Boletim Técnico de Solos, 5)

ZHANG, J . \& BARBER, S.A. Mayze root distribution between phosphorus-fertilized and unfertilized soil. Soil Sci. Soc. Am. J., 56:819-822, 1992. 